\title{
O PSICÓlOGO NO AMBIENTE HOSPITALAR: TÉCNICAS COGNITIVO-COMPORTAMENTAIS PARA A REDUÇÃO DO ESTRESSE E ANSIEDADE
}

\section{ARTIGO DE REVISÃO}

RIBEIRO, Thayanne Mayul de Souza Borges ${ }^{1}$

RIBEIRO, Thayanne Mayul de Souza Borges. O Psicólogo no ambiente hospitalar: técnicas cognitivo-comportamentais para a redução do estresse e ansiedade. Revista Científica Multidisciplinar Núcleo do Conhecimento. Ano 05, Ed. 08, Vol. 06, pp. 05-14. Agosto de 2020. ISSN: 2448-0959, Link de acesso: https://www.nucleodoconhecimento.com.br/psicologia/estresse-e-ansiedade

\section{RESUMO}

Este artigo tem por finalidade expor algumas das principais técnicas fundamentadas na Terapia Cognitivo-Comportamental. Objetiva-se, apresentar as práticas para lidar e até mesmo reduzir com o estresse e a ansiedade dentro do contexto hospitalar. As intervenções mostram práticas como a respiração diafragmática, técnicas de relaxamento, treinamento do controle do estresse, registro de pensamentos disfuncionais que propiciam a reestruturação cognitiva. Realizou-se um levantamento de periódicos nacionais e internacionais acerca das técnicas utilizadas e, as consequências do estresse a ansiedade. $O$ modo como o sujeito se relaciona, interpreta e dá sentido e significado aos fatos e a situações estressoras, principalmente no ambiente hospitalar. Assim sendo, o psicólogo é o profissional responsável por facilitar à compreensão do processo saúde e doença. Além de dar suporte emocional a equipe multidisciplinar, aos pacientes e aos acompanhantes

\footnotetext{
${ }^{1}$ Especialização em Terapia Cognitiva-Comportamental pela Faculdade Brasileira de Ensino Pesquisa e Extensão (FABEX). Pós-graduação em Saúde Mental e Atenção Psicossocial pela Universidade Estácio de Sá. Licenciatura em Pedagogia Instituto Superior de Educação da Faculdade Fortium Brasília-DF.
} 
deles. Visto que a Terapia Cognitiva-Comportamental pode ser considerada com uma autoterapia que propicia o bem estar físico e psíquico.

Palavras-chave: estresse, ansiedade, ambiente hospitalar, técnicas cognitivocomportamentais.

\section{INTRODUÇÃO}

Atualmente o estresse e ansiedade tem se tornado um fenômeno mundial, de acordo com Ganster e Rosen (2013) mencionados por Ferreira et al. (2016). Uma experiência que se vivenciada durante um longo tempo pode gerar mudanças que podem vir a afetar a saúde física, mental e emocional.

Nesta perspectiva alguns profissionais que compõem a equipe multidisciplinar consideram as tarefas realizadas dentro do espaço hospitalar estressantes. Os profissionais estão em contato frequente com os pacientes que apresentam diversas patologias. O risco de contaminação aumenta quando se entra em contato com aqueles que foram submetidos a procedimentos invasivos (MATURANA E VALLE, 2014).

Desse modo o presente artigo tem por objetivo identificar de forma geral, algumas técnicas fundamentadas na Terapia Cognitivo-Comportamental. Dentre essas intervenções estão: a respiração diafragmática; técnicas de relaxamento; treinamento do controle do estresse; registro de pensamentos disfuncionais que possibilitam que haja a reestruturação cognitiva. Transformações na forma de pensar e posteriormente, de se comportar frente as situações (MARQUES e DELFINO, 2016). As seguintes questões que nortearam este trabalho: De que modo as técnicas fundamentadas na Terapia Cognitiva-Comportamentais podem auxiliar na redução do estresse e da ansiedade no ambiente hospitalar? O uso dessas práticas pode vir a propiciar o bem estar físico e psíquico?

Realizou-se uma revisão de literatura por meio de um levantamento, com abordagem qualitativa, da publicação de artigos nacionais e internacionais acerca da temática. 
Existentes na base de dados da Scielo e do Google acadêmico. Os principais pesquisadores estudados para a elaboração desse material foram Gazzaniga e Heatherton (2005), Marques e Delfino (2016), Franco Adriano et al. (2017), Lima; Silva e Souza (2019), Mandato (2019), Yokoyama (2019), entre outros.

Este tema é relevante, pois o tempo no ambiente hospitalar pode ser determinante. $E$ isso requer a utilização de técnicas mais breves e diretivas. Pois, possibilitam ao sujeito lidar com as situações estressoras, decorrentes da rotina e das condições impostas pelo próprio contexto em que se encontra (PERON e SARTES, 2015).

\section{EMOÇÕES A ANSIEDADE E O ESTRESSE}

As emoções dão significado e sentido à vivência humana. Alertam sobre o perigo, formam laços afetivos entre os indivíduos. Contudo podem ocasionar alguns problemas. Uma pessoa muito ansiosa pode se ver paralisada diante de uma situação nova (GAZZANIGA e HEATHERTON, 2005).

De acordo com Gazzaniga e Heatherton (2005) os especialistas psicológicos acreditam que as emoções são respostas rápidas e automáticas aos eventos ambientais e estão ligadas diretamente aos sentimentos. Os autores ainda diferenciam a emoção do humor. Este reflete os estados emocionais diversos e permanentes que exercem influência de modo negativo ou positivo sobre o pensamento e o comportamento da pessoa. Considerado como um padrão de respostas comportamentais e fisiológicas que extrapolam as capacidades do organismo.

O quão estressada a pessoa se sente é determinada por diversos: a maneira como ela percebe a condição estressora e as crenças subjetivas acerca dos recursos internos que ela possui para agir diante do evento estressor. Um estímulo ameaçador do meio, no qual está inserido que requer uma resposta imediata no intuito de reduzir ou até mesmo fugir do estímulo encarado como ameaçador (GAZZANIGA e HEATHERTON, 2005). 
Da perspectiva evolutiva, a ansiedade e o estresse tem a origem no mecanismo de defesa do organismo. Ao se deparar com uma situação perigosa acontece um conjunto de respostas tanto comportamentais quanto neurovegetativas que correspondem à reação de medo (MARGIS et al., 2003). O sistema nervoso neurovegetativo corresponde ao sistema que envolve todos os nervos e centro nervosos que comandam as vísceras e as glândulas do corpo. Participando de todas a todas as funções do organismo (SOBRINHO, 2003). A ansiedade pode ser vista como uma resposta de maneira inconsciente ao estresse. Todavia o agente causador do estresse não é conhecido (BACHION et al., 1998)

Todas as pessoas vivenciam algum nível de ansiedade no dia a dia. Uma sensação de que algo está para acontecer. Mas, o sujeito não consegue identificar a causa do perigo, não consegue identificar o objeto de ameaça. Durante o estágio adaptativo, a ansiedade diminui o estado de tensão. $\mathrm{E}$ há diferentes graus do mais leve que deixa o indivíduo física e mentalmente mais atento. Até o grau mais elevado, no qual se sente paralisado. Tornando-o improdutivo e esse estado pode se cronificar e propiciar o surgimento de doenças (BACHION et al., 1998).

Conforme Franco Adriano et al. (2017) o estresse possui componentes físicos, fisiológicos e emocionais. Um comportamento que o organismo apresenta frente a uma circunstância que pode ser interpretada de forma negativa ou positiva. Esta última acontece no estágio inicial conhecido como fase de alerta. O sujeito consegue se esquivar e fugir das situações vistas como ameaçadoras. Entretanto se a situação estressante permanecer por um longo tempo a pessoa começa sentir um cansaço ao extremo.

O estágio posterior é a da semi-exaustão a tensão extrapola o limite daquilo que é possível controlar. A exaustão é o período mais negativo há um desequilíbrio interno o indivíduo se sente incapaz de tomar decisões, se concentrar nas tarefas diárias As patologias começam a aparecer após uma diminuição da resistência física e emocional do organismo. (FRANCO ADRIANO et al., 2017) 
Segundo Metring (2011) o Sistema Nervoso Autônomo (SNA) é formado por duas divisões: os sistemas Parassimpático (P) e Simpático (S), cujas funções opostas possibilitam a homeostase, o equilíbrio do sistema biológico mesmo em situações de alterações constantes do ambiente. O sistema parassimpático age de maneira mais conservadora, já o sistema simpático prepara o organismo para responder de forma imediata a circunstâncias consideradas estressantes.

Gazzaniga e Heatherton (2005) mencionam o termo resposta de luta-ou fuga criado pelo psicólogo Walter Cannon, de Harvard, para descrever a reações físicas dos animais diante de um ataque. $O$ baço se contrai e os batimentos cardíacos aumentam redistribuindo o sangue da pele e das vísceras para a musculatura e cérebro. A respiração fica ofegante, já as pupilas dos olhos se dilatam. O fígado libera uma enorme quantidade de glicose no organismo. $E$ isto acaba liberando uma grande quantidade de energia. Tudo acontece em segundos e possibilita direcionar toda a energia ao enfrentamento da ameaça.

Ganster e Rosen (2013) citado por Ferreira et al. (2016) ressalta que o estresse no ambiente de trabalho tem se tornado um fenômeno mundial, em longo prazo podem gerar alterações que afetam a saúde física, mental e emocional dos profissionais.

Alguns profissionais da saúde podem considerar as tarefas realizadas no hospital como estressantes. Uma organização com estrutura hierarquizada, regras rígidas de funcionamento, na qual o trabalhador entra em contato constante com pacientes que apresentam as mais diversas doenças (STEKEL, 2011). Um local onde risco de contaminação é frequente, exigindo atenção maior por parte do trabalhador. Principalmente, quando este está em contato com os que realizaram procedimentos invasivos (MATURANA E VALLE, 2014).

\section{O PAPEL DO PSICÓLOGO NO ESPAÇO HOSPITALAR}

O campo da saúde requer dos trabalhadores diariamente uma resposta imediata de maneira que a assistência dada aos pacientes possibilite minimizar as consequências. Essencialmente, no que se refere à negligência das condutas, a evitar os erros e a 
exposição às situações agravantes. Associadas a complexidade das atividades realizadas, o grau de comprometimento e as responsabilidades impostas por este tipo de atendimento que exige contato permanente com o sofrimento dos outros. Isso aumenta ainda mais a ansiedade e o estresse dos pacientes, acompanhantes deles e das equipes multidisciplinares de saúde que atuam dentro deste contexto (FRANCO ADRIANO et al., 2017).

Nesta perspectiva, a Psicologia Hospitalar pode ser vista como um campo com múltiplas possibilidades de atuação (ALMEIDA e MALAGRIS, 2011). Nos mais diversos setores desde o ambulatório até nas unidades de terapia intensiva (UTIS). Nas enfermarias integra as equipes responsáveis pelos cuidados paliativos, especialmente daqueles que se encontram na fase de finitude da vida. O psicólogo no ambiente hospitalar trabalha de forma interdisciplinar, com o intuito de tornar a atuação das equipes multidisciplinares de saúde mais humanizadas (LIMA, SILVA e SOUZA, 2019).

O psicólogo hospitalar intermedia as relações entre o paciente, à família dele e a equipe de saúde. O profissional responsável por dar voz às pessoas fragilizadas. Por meio de uma escuta acolhedora, na qual é possível compreender os medos à insegurança que a internação provoca. $E$ do uso de práticas interventivas que possibilitem reduzir os níveis de estresse, a ansiedade diante das situações vivenciadas (LIMA; SILVA e SOUZA, 2019).

O interesse pela atuação neste campo aparece da necessidade de se compreender e refletir sobre a saúde e a doença em uma perspectiva psicossocial e da intervenção individual e/ ou grupal (ALMEIDA e MALAGRIS, 2011). Nesse contexto o uso da Terapia Cognitiva Comportamental torna-se muito benéfico, pois o hospital é um ambiente que requer a utilização de técnicas mais diretivas, modalidade breve direcionada para criação de mecanismo que permitam o sujeito enfrentar a condição de adoecimento e de trabalho impostas (NEVES NETO, 2001 apud PERON e SARTES, 2015). 


\section{O PSICÓLOGO NO HOSPITAL E AS INTERVENÇÕES COGNITIVA- COMPORTAMENTAIS}

O psicólogo facilita a compreensão do processo saúde e doença, dando suporte emocional aos profissionais da saúde, aos pacientes e aos acompanhantes deles. No entanto, o tempo é um fator determinante que delimita a atuação do psicólogo dentro do espaço hospitalar e as questões a serem trabalhadas devem estar centradas no presente (YOKOYAMA, 2019).

De acordo com Judith Beck a Terapia Cognitiva-Comportamental surgiu no começo da década de 1960, por Aaron T. Beck, como psicoterapia breve e estruturada. Focada no momento atual na resolução dos problemas que se apresentam aqui e agora, na mudança dos pensamentos e nos comportamentos disfuncionais (BECK, 1997).

Portanto, uma psicoterapia educativa centrada no presente e no que motivou a procurar um tratamento. A metodologia utilizada fundamenta-se na cooperação entre psicoterapeuta e o cliente, de modo a provocar as mudanças necessárias que o permitam superar os problemas atuais. Os propósitos estabelecidos com base nas questões apresentadas propiciam à pessoa a aprendizagem de novas estratégias consideradas mais funcionais (LIMA, 2013).

Os programas para manter a ansiedade e o estresse, sob controle começaram a ser aplicados em meados da década de 1970 (MURTA, 2005). Entretanto no Brasil a Terapia Cognitiva-Comportamental (TCC) começou a se desenvolver no final dos anos de 1980. Desde então a utilização vem aumentando gradualmente, sobretudo nos últimos anos (PERON e SARTES, 2015). As Intervenções CognitivaComportamentais fundamentam-se na compreensão subjetiva de cada indivíduo por meio da tríade dos pensamentos (a maneira como ele vê a si mesmo, o meio e projeta o futuro), gerados por ele. Observa-se que os pensamentos (crenças) formam as emoções e essas originam o comportamento (MARQUES e DELFINO, 2016).

Atualmente, de acordo com Murta (2005), a maioria das estratégias para a redução de estresse e ansiedade é centrada no sujeito com intervenções multimodais, das 
quais a terapia cognitivo-comportamental vem ganhando cada vez mais destaque. $\mathrm{O}$ sujeito passa a ter conhecimento a respeito das causas e consequências do estresse e da ansiedade.

As intervenções cognitivo-comportamentais buscam diversificar o uso de técnicas que permitem o estabelecimento de um repertório mais saudável diante das circunstâncias que desencadeiam a ansiedade e o estresse exacerbados (MURTA, 2005). Algumas incluem as práticas das técnicas de respiração diafragmáticas, de relaxamento e o treinamento do estresse. Além, dos registros de pensamentos disfuncionais, mindfulness e da reestruturação cognitiva (MARQUES e DELFINO, 2016).

$\mathrm{Na}$ respiração diafragmática a pessoa aprende a inspirar e a expirar por meio do diafragma. Solicita-se que ela coloque uma das mãos na região do abdômen e a outra na região do tórax. Sustentando a respiração dessa forma por aproximadamente três minutos. É possível reduzir a ansiedade ao se concentrar a atenção de modo consciente para o processo da respiração (MARQUES e DELFINO, 2016). As práticas de relaxamento possibilitam a pessoa atingir um estado de tranquilidade mental e físico. O organismo retorna ao estado normal por meio das atividades de contração e relaxamento, trabalhando a tensão muscular corporal (MANDATO, 2019).

O Treinamento que visa controlar o Estresse mais conhecido como (TCS) tem por finalidade propor uma mudança com relação no modo de vida e os comportamentos embasados nos quatro pilares que sustentam a técnica: a reestruturação cognitiva e comportamental; práticas de atividades físicas; nutrição mais saudável; além das técnicas de relaxamento que reduzem a tensão física e mental (BORGES et al., 2009). $\mathrm{Na}$ técnica de mindfulness o indivíduo foca no momento atual ao registrar as emoções, os pensamentos e os comportamentos. Leva a atenção de modo sustentado a cada tarefa realizada ou fenômeno vivenciado (MARQUES e DELFINO, 2016).

Conforme Mandato (2019) nos registros de pensamento disfuncionais o sujeito consegue verificar os pensamentos menos adaptativos que causam o sofrimento. A partir da reestruturação cognitiva aprende a observar e identificar os pensamentos disfuncionais substituindo-os por mais adaptativos e consequentemente há uma 
mudança no comportamento (LIMA; SILVA e SOUZA, 2019). Borges et al. (2009) enfatizam que o enfrentamento de circunstâncias estressoras por meio da estratégia de reestruturação cognitiva reduz os níveis de estresse e ansiedade.

Stekel (2011) afirma que por meio desta terapia o sujeito torna-se capaz de lidar de maneira mais funcional com esses estímulos estressores a partir do momento que compreende o meio no qual está inserido. Por seu caráter educativo, a Terapia Cognitiva-Comportamental possibilita ao cliente realizar uma autoterapia.

Dado que visa ensinar a pessoa algumas técnicas que possibilitam prevenir recaídas e promover o bem estar físico e mental (MANDATO, 2019). Pretende-se diminuir os níveis de estresse e da ansiedade no organismo. E consequentemente melhorar qualidade de vida dos pacientes, acompanhantes desses e dos profissionais que compõe a equipe multidisciplinar de saúde (LIMA; SILVA e SOUZA, 2019).

\section{CONSIDERAÇÕES FINAIS}

As atividades ocupacionais podem ser consideradas por muitas vezes como uma fonte de realizações. Entretanto muitas vezes a pessoa se sente incapaz de lidar com as pressões que a rotina ocupacional impõe. Assim sendo, alguns profissionais de saúde podem sentir-se exaustos durante o trabalho. Pois realizam no dia a dia uma grande quantidade de atendimento e estão em contanto frequente com os mais diversos tipos de patologias. E isso acaba aumentando o estresse e a ansiedade. Portanto, devem atentar-se à saúde física e psíquica. Sobretudo, devido à particularidade das tarefas que desempenham.

Verifica-se que o trabalho dos psicólogos que utilizam a Terapia CognitivaComportamental no ambiente hospitalar tem crescido consideravelmente nas últimas décadas. Focada no momento atual, esse tipo de psicoterapia breve e estruturada tem se mostrado muito benéfica sob esta perspectiva. A abordagem cognitivacomportamental associa técnicas cognitivas e comportamentais. 
A utilização dessas práticas possibilita desenvolver as competências necessárias para que a pessoa consiga lidar da melhor maneira com os estímulos estressores presentes no ambiente, sobretudo o hospitalar. Dessa forma, o artigo em questão pode ser considerado como base para que sejam realizadas novas pesquisas possam ampliar a discussão e a compreensão acerca dessa temática. Pois, essa terapia pode vir a auxiliar maneira significativa na redução da ansiedade e do estresse.

\section{REFERÊNCIAS}

ALMEIDA, R.; MALAGRIS, LM. A prática da psicologia da saúde Revista Sociedade Brasileira de Psicologia Hospitalar v. 14, n.2, p.183-202, 2011. Disponível em: $<$ <ttp://pepsic.bvsalud.org/scielo.php?script=sci_arttext\&pid=S1516$08582011000200012>$ Acesso em: 13 Mai. 2020.

BACHION M M., et al. ESTRESSE, ANSIEDADE E COPING: Uma revisão dos conceitos, medidas e Estratégias de Intervenção Voltadas para Prática de Enfermagem. Revista Mineira de Enfermagem, v. 2, n.1, p. 33-39, 1998. Disponível em:<http://www.reme.org.br/artigo/detalhes/867> Acesso em: 22 Mai. 2020.

BECK J.S. Pontos a serem problematizados e discutidos: História da Terapia Cognitiva e os dez princípios da Terapia Cognitiva elencados por Judith Beck. Terapia Cognitiva: teoria e prática. Porto Alegre: Artmed, pp.17-25, 1997.

BORGES C., et al. Intervenção cognitivo-comportamental em estresse e dor crônica. Arquivos Ciências Saúde, v.16, n.4, p.181-186, 2009. <http://repositorioracs.famerp.br/racs_ol/vol-16-4/IDK7_out-dez_2010.pdf> Acesso em: 09 Jun. 2020.

FERREIRA CA., et al. O contexto do estresse ocupacional dos trabalhadores da saúde: Estudo Bibliométrico. Revista de Gestão em Sistemas de Saúde. v.5, n.2, p. 84-99, 2016. Disponível em: <http://www.revistargss.org.br/ojs/index.php/rgss/article/view/233> Acesso em: 24 Mai.2020. 
FRANCO ADRIANO M, et al. Estresse Ocupacional em Profissionais da Saúde que atuam no Serviço de Atendimento Móvel de Urgência de Cajazeiras-PB. Revista Brasileira de Ciências da Saúde, v. 21, n.1, p. 29-34, 2017. Disponível em:<https://periodicos.ufpb.br/index.php/rbcs/article/view/16924> Acesso em: 30 Abr.2020.

GAZZANIGA, M.; HEATHERTON, T. Emoções, Estresse e Coping. Maria Adriana Veronese (trad.). Ciência Psicológica Mente, Cérebro e Comportamento. 2. ed.Porto Alegre: Artmed, pp.312-341, 2005.

LIMA A. Terapias Cognitivo-Comportamentais. Técnicas Psicoterápicas/ Séries Concursos Públicos: Resumo de Psicologia. Salvador: Concursos PSI Empreendimentos Editoriais, pp.42-45, 2013.

LIMA, F.; SILVA, AC.; SOUZA T. Olhar Humanizado na Prática do Psicólogo no Ambiente Hospitalar. GEPNEWS, Maceió, a.3, v.2, n.2 pp.448-453, 2019.

MANDATO, T. Contribuições da Terapia Cognitivo-Comportamental no Tratamento da Síndrome de Burnout em Profissionais da Saúde. Trabalho de Conclusão (Especialização Terapia Cognitivo-Comportamental). Centro de Estudos em Terapia Cognitivo-Comportamental. São Paulo, 36 p. 2019.

MARGIS R, et al. Relação entre estressores estresse e ansiedade. Revista de Psiquiatria, v.25 (suplemento 1), p 65-74, 2003. Disponível em: $<$ https://www.scielo.br/scielo.php?pid=S0101-

81082003000400008\&script=sci_abstract\&tlng=pt >Acesso em: 28 Abr. 2020.

MARQUES L, DELFINO TE. Contribuições das Técnicas de Respiração, Relaxamentos e Mindfulness no manejo do estresse ocupacional. PSICOLOGIA. PT O Portal dos Psicólogos, 2016. <https://www.psicologia.pt/artigos/textos/A0965.pdf> Acesso em: 10 Mai. 2020.

MATURANA, AP.; VALLE, TG. Estratégias de Enfrentamento de Situações Estressoras de Profissionais no Ambiente Hospitalar. Revista Psicologia Hospitalar, 
v.12,

n.2,

pp.2-23,

2014.

Disponível

em:

<http://pepsic.bvsalud.org/scielo.php?script=sci_arttext\&pid=S1677-

74092014000200002> Acesso em: 27 Abr. 2020.

METRING R. Como se organiza o Sistema Nervoso? Neuropsicologia e aprendizagem: fundamentos necessários para o planejamento do ensino. Rio de Janeiro: Wak, 110p.

MURTA, S. Programas de manejo de estresse ocupacional: uma revisão sistemática da literatura. Revista Brasileira de Terapia Comportamental, VII, n.2, p. 159-177, 2005.

Disponível

em:

<http://pepsic.bvsalud.org/scielo.php?script=sci_arttext\&pid=S1517-

55452005000200003> Acesso em: 10 Mai. 2020.

PERON, N.; SARTES, L. Terapia cognitivo-comportamental no hospital geral: revisão da literatura brasileira. Revista Brasileira de Terapias Cognitivas, v. 11, n.1, p. 4249 2015.

Disponível em: <http://pepsic.bvsalud.org/scielo.php?script=sci_arttext\&pid=S180856872015000100006> Acesso em: 28 Mai. 2020.

SOBRINHO, J. Fisiologia do sistema nervoso neurovegetativo. Revista ACTA FISIÁTRICA, v. $10, \quad$ n.3, p. 122-132, 2003. Disponível em: <http://www.revistas.usp.br/actafisiatrica/article/view/102458> Acesso em: 10 Jun. 2020.

STEKEL, L. Estresse e Coping entre auxiliares e técnicos de enfermagem de um hospital universitário. 2011.110f. Dissertação (Mestrado em Enfermagem). Universidade Federal de Santa Maria, Santa Maria, 2011.

YOKOYAMA, F. Aplicação da Terapia Cognitivo-Comportamental no Contexto Hospitalar. Trabalho de Conclusão (Especialização Terapia CognitivoComportamental). Centro de Estudos em Terapia Cognitivo-Comportamental. São Paulo, 25 p. 2019. 
Enviado: Julho, 2020.

Aprovado: Agosto, 2020. 